\title{
DYNAMIC PORTFOLIO SELECTION \\ IN A DUAL EXPECTED UTILITY THEORY FRAMEWORK
}

\author{
BY \\ Marisa Cenci, Massimiliano Corradini and Andrea Gheno*
}

\begin{abstract}
In this paper the dynamic portfolio selection problem is studied for the first time in a dual utility theory framework. The Wang transform is used as distortion function and well diversified optimal portfolios result both with and without short sales allowed.
\end{abstract}

\section{KEYWORDS}

Dynamic Portfolio Selection, Dual Utility Theory, Merton Problem, Wang Transform.

\section{INTRODUCTION}

In this paper a new approach for solving the dynamic portfolio selection problem, also known as the Merton (1969) problem, is introduced. This approach is based on the dual expected utility (DEU) theory which is a particular class of non-expected utility theory presented in Yaari (1987). Unlike the classical expected utility (EU) theory by von Neumann and Morgenstern (1944) the DEU theory overcomes some paradoxes such as Allais (1953) and Ellsberg (1961), as shown in Quiggin (1993).

In the DEU framework "attitudes toward risks are characterized by a distortion applied to probability distribution functions, in contrast to expected utility in which attitudes toward risks are characterized by a utility function of wealth" (Wang-Young (1998)).

As far as the authors know in financial and economic literature there is not any work concerned with the application of the DEU theory to dynamic selection of an asset portfolio. This may be due to the fact that in Yaari (1987) it is shown that DEU theory leads to not diversified portfolios when the decision maker has only two assets available, one risky and one not. However in HadarKun Seo (1995) it is shown that in the presence of many risky assets DEU theory

* The authors are from the Department of Economics, Università degli studi Roma Tre, Rome, Italy. 
is consistent with optimal well diversified portfolios. This justifies a DEU approach to the solution of the Merton (1969) problem which in this paper is considered with and without trading strategy constraints. However, in a very recent article Hamada et al. (2006) set out a general layout for portfolio optimization in a discrete time setting, consistent with the DEU theory.

In this paper an application is also proposed where the Wang transform is chosen as the distortion function. In particular it is shown that the unconstrained optimization problem admits solutions only under adequate hypotheses. Conversely it is shown that the constrained problem, i.e. without short-selling allowed, always admits a solution in the feasible region.

The remainder of the paper is structured as follows. In section 2 outlines of the EU and DEU theories are given. In section 3 the financial model which is used in section 4 to study the dynamic portfolio selection problem with DEU theory is described. In section 5 the Wang transform is applied to a portfolio selection problem with one risk-free and several risky assets available. In the final section the conclusions are drawn.

\section{EU THEORY vs DEU THEORY}

Today the EU theory by von Neumann and Morgenstern (1944) is still the most popular approach to problems of decision making under uncertainty, however since the sixties several authors have empirically shown that actual decisions are not always consistent with all EU theory axioms.

If with $\chi$ is denoted the opportunity set and with capital letters the opportunities which can be degenerate or not degenerate random variables, the EU theory axioms are:

A.1) Completeness $-\forall X$ and $Y \in \chi$ it is either $X \succeq Y$ or $X \preceq Y$

A.2) Transitivity - if $X \succeq Y$ and $Y \succeq Z \Rightarrow X \succeq Z$

A.3) Continuity - if $X \succeq Y \succeq Z \Rightarrow \exists p \in[0,1]: Y \sim p X+(1-p) Z$

A.4) Independence - if $X \succeq Y \Rightarrow \forall p \in[0,1] p X+(1-p) Z \succeq p Y+(1-p) Z$

Axioms A.1, A.2, A.3 always hold while the independence axiom, as also shown in Allais (1953) and Ellsberg (1961), is violated. In order to avoid this problem several alternative utility theories, called non-expected utility theories, have been presented in the literature. The DEU theory is a non-expected utility theory whose axioms are A.1, A.2, A.3 and

A.4*) Comonotonicity - if $X, Y, Z$ are pairwise comonotonic ${ }^{1}$ and $X \succeq Y$

$$
\Rightarrow \forall p \in[0,1] p X+(1-p) Z \succeq p Y+(1-p) Z
$$

\footnotetext{
${ }^{1} X$ and $Y$ are comonotonic if there exist a random variable $Z$ and two not decreasing real functions $f$ and $h$ such that $X=f(Z)$ and $Y=h(Z)$.
} 
Under axioms A.1, A.2, A.3 and A.4* in Yaari (1987) it is shown that a nondecreasing function $g:[0,1] \rightarrow[0,1]$, with $g(0)=0$ and $g(1)=1$ exists such that $X \succeq Y$ if and only if

$$
\int_{-\infty}^{+\infty} x d g\left(F_{X}(x)\right) \geq \int_{-\infty}^{+\infty} y d g\left(F_{Y}(y)\right)
$$

where $F_{X}(x)$ and $F_{Y}(y)$ are the probability distribution functions of the random variables $X$ and $Y$.

In Wang-Young (1998) it is shown how the function $g$ affects the probability distribution functions of the random variables considered.

The analytical form of $g$ embeds the degree of aversion towards risk of the decision maker. In particular in Quiggin (1993) it is shown that a concave and increasing function $g$ characterizes a risk-averse decision maker and the resulting ordering is consistent with the first and second order stochastic dominance principles.

If the dual expected utility of the random variable $X$ is denoted with

$$
\operatorname{DEU}(X) \equiv E_{g}[X]=\int_{-\infty}^{+\infty} x d g\left(F_{X}(x)\right)
$$

the following properties hold²:

P.1) if $g(F(x))=F(x), D E U(X)=E(X)$

P.2) $D E U(a X+b)=a D E U(X)+b \quad \forall a>0, b \geq 0$

P.3) if $X$ and $Y$ are comonotonic $D E U(X+Y)=D E U(X)+D E U(Y)$

P.4) if $g$ is concave $D E U(X) \leq E(X)$ and $D E U(X+Y) \geq D E U(X)+D E U(Y)$

\section{Financial Market Model}

\subsection{The Assets}

A financial market where $n$ risky assets and a risk-free asset are traded is considered. At time $s \in[t, T]$ the market prices of risky assets and that of the risk-free asset are respectively $\left\{P_{i}(s), i=1, \ldots, n\right\}$ and $P_{0}(s)$.

The risk source is represented through a standard Wiener process $W=\left(W_{1}\right.$, $\left.\ldots, W_{n}\right)^{*}$ in $\mathbb{R}^{n}$, with $W_{i}$ not correlated with $W_{j}$ for $i \neq j$, which affects the evolution of risky asset prices by the following stochastic differential equations:

$$
d P_{i}(s)=P_{i}(s)\left(\mu_{i}(s) d s+\sum_{j=1}^{n} \sigma_{i j}(s) d W_{j}(s)\right), i=1, \ldots, n
$$

2 For more details see Wang-Young (1998). 
where $\mu_{i}(s)$ is the instantaneous return rate of the $i$-th risky asset and $\left\{\sigma_{i j}(s)\right.$, $s \in[t, T]\}$ is the volatility matrix.

The deterministic evolution of the risk-free asset is:

$$
d P_{0}(s)=P_{0}(s) r(s) d s
$$

where $r(s)$ is the instantaneous risk-free rate and $P_{0}(0)=1$. In the remainder of the paper it is obviously assumed that $\mu_{i}(s)>r(s), \forall s \in[t, T]$.

As it is usually done, it is also assumed that, $\forall s \in[t, T]$ :

H.1) the processes $r(s), \mu(s)=\left(\mu_{1}(s), \ldots, \mu_{n}(s)\right)^{*}$ and the matrix $\sigma(s)=\left\{\sigma_{i j}(s)\right\}$ are adapted to the filtration $\mathcal{F}_{s}=\sigma(W(u), u \in[t, s])$

H.2) $\sigma(s)$ is not degenerate in strong form so that if $D(s)=\sigma^{*}(s) \sigma(s), \forall \varepsilon>0$ : $\xi^{*} D(s) \xi \geq \varepsilon\|\xi\|^{2}$ a.s. $\forall(s, \xi) \in[t, T] \times \mathbb{R}^{n}$

H.3) $r(s) \geq-\eta, \eta>0$

\subsection{The Portfolio}

At time $t$ a price-taker agent $I$ with an initial wealth $X(t)=x>0$ is considered. At every time $s \in[t, T]$ the agent $I$ selects the quantity of each risky asset $\left(\Phi_{1}(s)\right.$, $\left.\ldots, \Phi_{n}(s)\right)^{*}$ and the quantity of the risk-free asset $\Phi_{0}(s)=X(s)-\sum_{i=1}^{n} \Phi_{i}(s) P_{i}(s)$ to hold over the infinitesimal time interval $[s, s+d s)$.

Hence the trading strategy of $I$ is represented by the process $\left(\Phi_{0}(s), \ldots, \Phi_{n}(s)\right)^{*}$ which is assumed to be adapted to the current information $\mathcal{F}_{s}$ and such that $\int_{t}^{T}\left[\Phi_{i}(s)\right]^{2} d s<+\infty \forall i=1, \ldots, n$ a.s. In order to have a self-financing trading strategy the following relation must hold:

$$
\sum_{i=0}^{n} \Phi_{i}(s) P_{i}(s)=X(t)+\sum_{i=0}^{n} \int_{t}^{s} \Phi_{i}(u) d P_{i}(u), \forall s \in[t, T]
$$

or, in differential form,

$$
d \sum_{i=0}^{n} \Phi_{i}(s) P_{i}(s)=\sum_{i=0}^{n} \Phi_{i}(s) d P_{i}(s), \quad \forall s \in[t, T] .
$$

Under these hypotheses the wealth of the agent $I$ at time $s$ is:

$$
X(s)=\sum_{i=0}^{n} \Phi_{i}(s) P_{i}(s)
$$

where

$$
\Phi_{i}(s)= \begin{cases}\frac{X(s) \pi_{i}(s)}{P_{i}(s)} & i=1, \ldots, n \\ \frac{X(s)\left[1-\sum_{i=1}^{n} \pi_{i}(s)\right]}{P_{0}(s)} & i=0\end{cases}
$$


and the portfolio process is:

$$
\pi(s)=\left(\pi_{1}(s), \ldots, \pi_{n}(s)\right)^{*} .
$$

The resulting wealth evolution can be expressed through the following relation ${ }^{3}$ :

$$
d X(s)=X(s)[r(s)+\hat{\mu}(s) \cdot \pi(s)] d s+X(s) \pi(s) \cdot \sigma(s) d W(s)
$$

with $X(t)=x$ and $\hat{\mu}_{i}=\mu_{i}-r$.

In the remainder of the paper the solution of equation (1) relative to the portfolio process $\pi$ and initial condition $X(t)=x$ is denoted with $X_{s}^{t, x, \pi}, s \in$ $[t, T]$ and the associated conditional probability function of the final wealth $\operatorname{Prob}\left[X_{T}^{t, x, \pi} \leq y \mid X(t)=x\right]$ with $F_{t, x}^{\pi}(y)$.

\section{Dynamic Portfolio Selection by DeU Theory}

The unconstrained dynamic portfolio selection problem by DEU theory can be expressed as an optimal stochastic control problem whose control variables are identified by the vector $\pi(s)=\left(\pi_{1}(s), \ldots, \pi_{n}(s)\right)^{*}$ and the functional to be optimized is

$$
W^{\pi}(t, x) \equiv E_{g}\left[X_{T}^{t, x, \pi}\right]=\int_{-\infty}^{+\infty} y d g\left(F_{t, x}^{\pi}(y)\right)
$$

The optimal control is determined through the optimization of the functional $W^{\pi}(t, x)$ or, equivalently, by determining the function $v(t, x)$ such that

$$
v(t, x)=\sup _{\pi \in K} E_{g}\left[X_{T}^{t, x, \pi}\right]=\sup _{\pi \in K} \int_{-\infty}^{+\infty} y d g\left(F_{t, x}^{\pi}(y)\right),
$$

where $K$ is the feasible set, the stochastic evolution of $X_{T}^{t, x, \pi}$ is given by equation (1) and $v(T, x)=x$.

If, as in Karatzas-Kou (1996), it is assumed that

$$
X_{T}^{t, \lambda x, \pi}=\lambda X_{T}^{t, x, \pi}, \quad \lambda>0
$$

the following proposition is valid:

Proposition 4.1. The $v(t, x)$ function is proportional to the initial wealth $x$ : $v(t, x)=\alpha(t, \bar{\pi}) x$, where $\bar{\pi}$ satisfies equation (2).

Proof. Let $x$ and $x^{\prime}$ be two different values of the initial wealth, and let $\lambda=x^{\prime} / x$. The relation (3) can be rewritten as

$$
X_{T}^{t, x^{\prime}, \pi}=\frac{x^{\prime}}{x} X_{T}^{t, x, \pi}
$$

$\overline{3 \text { For } a, b \in \mathbb{R}^{n}}$ the inner product between the vector $a$ and $b$ is denoted with $a \cdot b \equiv \sum_{i=1}^{n} a_{i} b_{i}$. 
Given the initial investment $x^{\prime}$ the conditional distribution function of the final wealth is

$$
\begin{aligned}
F_{t, x^{\prime}}^{\pi}(y) & =\operatorname{Prob}\left[X_{T}^{t, x^{\prime}, \pi} \leq y\right] \\
& =\operatorname{Prob}\left[\frac{x^{\prime}}{x} X_{T}^{t, x, \pi} \leq y\right] \\
& =\operatorname{Prob}\left[X_{T}^{t, x, \pi} \leq \frac{x}{x^{\prime}} y\right] \\
& =F_{t, x}^{\pi}\left(\frac{x}{x^{\prime}} y\right) .
\end{aligned}
$$

Therefore

$$
\begin{aligned}
W^{\pi}\left(t, x^{\prime}\right) & =\int_{-\infty}^{+\infty} y d g\left(F_{t, x^{\prime}}^{\pi}(y)\right) \\
& =\int_{-\infty}^{+\infty} y d g\left(F_{t, x}^{\pi}\left(\frac{x}{x^{\prime}} y\right)\right)
\end{aligned}
$$

and with $y=\frac{x^{\prime}}{x} y^{\prime}$ it follows that

$$
\begin{aligned}
W^{\pi}\left(t, x^{\prime}\right) & =\int_{-\infty}^{+\infty} \frac{x^{\prime}}{x} y^{\prime} d g\left(F_{t, x}^{\pi}\left(y^{\prime}\right)\right) \\
& =\frac{x^{\prime}}{x} \int_{-\infty}^{+\infty} y^{\prime} d g\left(F_{t, x}^{\pi}\left(y^{\prime}\right)\right) \\
& =\frac{x^{\prime}}{x} W^{\pi}(t, x) .
\end{aligned}
$$

The last equation shows that for all $\pi$

$$
W^{\pi}\left(t, x^{\prime}\right)=\frac{x^{\prime}}{x} W^{\pi}(t, x) \Leftrightarrow \frac{W^{\pi}\left(t, x^{\prime}\right)}{x^{\prime}}=\frac{W^{\pi}(t, x)}{x},
$$

which holds if and only if the ratio $W^{\pi}(t, x) / x$ is independent of $x$ :

$$
W^{\pi}(t, x)=\alpha(t, \pi) x .
$$

Taking the supremum of the last equation gives

$$
v(t, x)=\alpha(t, \bar{\pi}) x .
$$

From Proposition 4.1., it can be inferred that, unlike the EU case, the Bellman equation is not useful to express $\pi$ as a function of the ratio $\frac{\partial v}{\partial x} / \frac{\partial^{2} v}{\partial x^{2}}$ since the denominator is null. Therefore the solution of the problem must be determined by solving a non-standard Bellman equation for every given distortion function $g(\cdot)$. The optimal strategy is found by maximizing the resulting functional $W^{\pi}(t, x)$ with respect to the variations in $\pi$. 
If short sales are not allowed the following bounds need to be added to the optimization problem:

$$
\pi_{i} \geq 0 \quad \forall i=0, \ldots, n
$$

and

$$
\sum_{i=1}^{n} \pi_{i}(t) \leq 1
$$

However regardless of the presence of these bounds the existence and uniqueness of the solution strongly depend on the functional form of the transformation $g(\cdot)$ chosen.

\section{DEU Theory AND the WANG TRANSForm}

In Wang (2000) and Wang (2002) a general framework for pricing financial and insurance risks is introduced. The methodology consists of using a particular class of distortion functions on the cumulative distribution function of the risky asset (or liability) future value. The resulting cumulative distribution function is "risk-adjusted" in the sense that its mean value discounted at the risk-free rate is equal to the current asset (liability) fair price. This approach is therefore consistent with the risk-neutral valuation framework commonly used in option theory. Denoting with $F_{X}(x)$ the probability distribution function of the random variable $X$ the Wang transform is:

$$
g\left(F_{X}(x)\right)=\Phi\left(\Phi^{-1}\left(F_{X}(x)\right)+\alpha\right) \alpha>0,
$$

where $\Phi$ is the normal cumulative distribution function

$$
\Phi(x)=\int_{-\infty}^{x} e^{-\frac{y^{2}}{2}} \frac{d y}{\sqrt{2 \pi}} .
$$

From equation (4) it is clear that the Wang transform is a horizontal translation of the probability distribution function. The effect of such translation is an overweighting of the left tail and an underweighting of the right one that overall leave the class of the distribution unchanged if the distribution $F_{X}(x)$ is normal.

In the remainder of this section it is assumed that equation (4) is the distortion function and that the stochastic evolution of the asset prices follows a geometric brownian motion with constant coefficients. The latter assumption is useful both for ease of computation and to overcome some peculiarities of the Wang transform.

The portfolio stochastic evolution is represented by equation (1) with constant coefficients:

$$
d X_{s}=X_{s}(r+\hat{\mu} \cdot \pi) d s+X_{s} \pi \cdot \sigma d W(s), s \in[t, T]
$$


with $X(t)=x$.

Therefore at time $T$ the wealth level $X_{T}^{t, x, \pi}$ is a lognormally distributed variable and $\ln X_{T}^{t, x, \pi}$ has a normal distribution with mean:

$$
m=\ln x+\left(r+\hat{\mu} \cdot \pi-\frac{\pi \cdot D \pi}{2}\right)(T-t),
$$

where $D=\sigma^{*} \sigma$, and variance:

$$
\Sigma^{2}=\pi \cdot D \pi(T-t) .
$$

Since the conditional distribution function $F_{t, x}^{\pi}(y)$ is

$$
F_{t, x}^{\pi}(y)=\int_{0}^{y} \frac{1}{y^{\prime} \sqrt{2 \pi \Sigma^{2}}} e^{-\frac{\left(\ln y^{\prime}-m\right)^{2}}{2 \Sigma^{2}}} d y^{\prime},
$$

with $\ln y^{\prime}=z$, the previous formula becomes

$$
\begin{aligned}
F_{t, x}^{\pi}(y) & =\int_{-\infty}^{\ln y} \frac{1}{\sqrt{2 \pi \Sigma^{2}}} e^{-\frac{(z-m)^{2}}{\Sigma^{2}}} d z \\
& =\Phi\left(\frac{\ln y-m}{\Sigma}\right)
\end{aligned} .
$$

Hence the transformed probability distribution function is

$$
\Phi\left(\Phi^{-1}\left(F_{t, x}^{\pi}(y)\right)+\alpha\right)=\Phi\left(\frac{\ln y-m}{\Sigma}+\alpha\right) .
$$

Thus the Wang transform of the conditional distribution final expected wealth is

$$
\begin{aligned}
E_{g}\left[X_{T}^{t, x, \pi}\right] & =\int_{0}^{+\infty} y d \Phi\left(\frac{\ln y-m}{\Sigma}+\alpha\right) \\
& =\int_{-\infty}^{+\infty} e^{z} d \Phi\left(\frac{z-m}{\Sigma}+\alpha\right)
\end{aligned}
$$

where $y=e^{z}$.

Substituting $\frac{z-m}{\Sigma}+\alpha=u$ into (5) gives

$$
\begin{aligned}
& E_{g}\left[X_{T}^{t, x, \pi}\right]=e^{m-\alpha \Sigma} \frac{1}{\sqrt{2 \pi}} \int_{-\infty}^{+\infty} e^{u \Sigma-\frac{u^{2}}{2}} d u= \\
& =e^{m-\alpha \Sigma+\frac{\Sigma^{2}}{2}}=x \exp \{(r+\hat{\mu} \cdot \pi)(T-t)-\alpha \sqrt{\pi \cdot D \pi} \sqrt{T-t}\} .
\end{aligned}
$$


Therefore

$$
\begin{aligned}
W^{\pi}(t, x) & =x \exp \{(r+\hat{\mu} \cdot \pi)(T-t)-\alpha \sqrt{\pi \cdot D \pi} \sqrt{T-t}\} \\
& =x e^{r(T-t)} e^{f(\pi)(T-t)}
\end{aligned}
$$

where

$$
f(\pi)=\hat{\mu} \cdot \pi-\beta \sqrt{\pi \cdot D \pi}
$$

with $\beta=\alpha / \sqrt{T-t}$. Thus the optimal trading strategy at time $t$ is found by solving the problem

$$
\sup _{\pi \in K}[\hat{\mu} \cdot \pi-\beta \sqrt{\pi \cdot D \pi}] \equiv \sup _{\pi \in K} f(\pi) .
$$

\subsection{The Unconstrained Case}

If short sales are allowed ${ }^{4} K=\mathbb{R}^{n}$ and the necessary conditions for solving the problem (7), if $\pi \neq 0$,

$$
\hat{\mu}_{i}-\beta \frac{\sum_{j=1}^{n} D_{i j} \pi_{j}}{\sqrt{\pi \cdot D \pi}}=0 \forall i=1, \ldots, n
$$

do not always admit solutions. In fact they admit solutions if and only if $\beta=$ $\sqrt{\hat{\mu} \cdot D^{-1} \hat{\mu}}$. In this case the optimal strategy is $\bar{\pi}=c D^{-1} \hat{\mu}$, with $c>0$ and the optimum value of (7) is

$$
\hat{\mu} \cdot \bar{\pi}-\beta \sqrt{\bar{\pi} \cdot D \bar{\pi}}=\hat{\mu} \cdot \bar{\pi}-\sqrt{\hat{\mu} \cdot D^{-1} \hat{\mu}} \sqrt{\bar{\pi} \cdot D \bar{\pi}}=0 .
$$

The strategy $\pi=0$ is also an optimum since $f(0)=0$. Therefore if $\beta=$ $\sqrt{\hat{\mu} \cdot D^{-1} \hat{\mu}}$ equation (7) admits the optimal strategy $\bar{\pi}=c D^{-1} \mu$, with $c \geq 0$. If $\beta<\sqrt{\hat{\mu} \cdot D^{-1} \hat{\mu}}$ the function $f(\pi)$ is unbounded, while for $\beta>\sqrt{\hat{\mu} \cdot D^{-1} \hat{\mu}}$ the function $f(\pi)$ is always non positive and reaches its optimum for $\pi=0$. Therefore the solution of the unconstrained problem is

$$
\sup _{\pi \in \mathbb{R}^{n}} f(\pi)= \begin{cases}0 & \text { if } \beta>\sqrt{\hat{\mu} \cdot D^{-1} \hat{\mu}} \rightarrow \bar{\pi}=0 \\ 0 & \text { if } \beta=\sqrt{\hat{\mu} \cdot D^{-1} \hat{\mu}} \rightarrow \bar{\pi}=c D^{-1} \hat{\mu}, c \geq 0 . \\ \nexists \quad \text { if } \beta<\sqrt{\hat{\mu} \cdot D^{-1} \hat{\mu}}\end{cases}
$$

$\overline{\forall \forall \pi \in \mathbb{R}^{n}: \pi} \cdot D \pi \geq 0$ since $D$ is positive definite. 
If $\beta \geq \sqrt{\hat{\mu} \cdot D^{-1} \hat{\mu}}$

$$
v(t, x)=\sup _{\pi \in \mathbb{R}^{n}} E_{g}\left[X_{T}^{t, x, \pi}\right]=x e^{r(T-t)},
$$

the optimal Wang distorted expectation $E_{g}\left[X_{T}^{t, x, \bar{\pi}}\right]$ is also the expectation under the unique risk-neutral measure $\mathbb{Q}^{5}$ :

$$
e^{-r(T-t)} \sup _{\pi \in \mathbb{R}^{n}} E_{g}\left[X_{T}^{t, x, \pi}\right]=e^{-r(T-t)} E^{\mathbb{Q}}\left[X_{T}^{t, x, \pi}\right] \equiv x,
$$

where the measure $\mathbb{Q}$ is generated by the Wiener process $W_{0}(s)$ given by

$$
d W_{0}(s)=d W(s)+\theta d s,
$$

with

$$
\theta_{i}=\sum_{j=1}^{n} \sigma_{i j}^{-1} \hat{\mu}_{j}
$$

and the corresponding portfolio evolution in a risk-neutral world is

$$
d X_{s}=r X_{s} d s+X_{s} \pi \cdot \sigma d W_{0}(s) .
$$

Introducing the market price of risk vector $q$

$$
\mu_{i}-r=\sum_{j=1}^{n} \sigma_{i j} q_{j}
$$

or, in vectorial form,

$$
\hat{\mu}=\sigma q \Leftrightarrow q=\sigma^{-1} \hat{\mu}
$$

it can be argued that the solution of problem (7) in the unconstrained case exists if and only if $\beta \geq\|q\|$.

\subsection{The Constrained Case}

If short sales are not allowed, in order to find the optimal strategy, the following constrained optimization problem must be solved:

$$
\sup _{\pi \in K}[\hat{\mu} \cdot \pi-\beta \sqrt{\pi \cdot D \pi}]
$$

5 See, for instance, Karatzas-Kou (1996). 
where $K$ is the $n$-dimensional simplex

$$
K=\left\{\pi \in \mathbb{R}^{n}: \sum_{i=1}^{n} \pi_{i} \leq 1, \pi_{i} \geq 0 \quad \forall i=1, \ldots, n\right\} .
$$

In this case the problem can be solved by the Kuhn-Tucker equation which in general does not admit analytical solutions.

If there are only two risky assets and a risk-free asset, the optimization problems (14-15) can be solved explicitly. In this case the feasible set $K$ is shown in figure 1.

On the frontier of the triangle $K$ there are the following suprema

$$
\begin{gathered}
\sup _{\pi \in A B} f(\pi)= \begin{cases}0 & \text { if } \hat{\mu}_{1}-\beta \sqrt{D_{11}}<0 \rightarrow \pi=(0,0)^{*} \\
\hat{\mu}_{1}-\beta \sqrt{D_{11}} & \text { if } \hat{\mu}_{1}-\beta \sqrt{D_{11}}>0 \rightarrow \pi=(1,0)^{*}\end{cases} \\
\sup _{\pi \in A C} f(\pi)= \begin{cases}0 & \text { if } \hat{\mu}_{2}-\beta \sqrt{D_{22}}<0 \rightarrow \pi=(0,0)^{*} \\
\hat{\mu}_{2}-\beta \sqrt{D_{22}} \text { if } \hat{\mu}_{2}-\beta \sqrt{D_{22}}>0 \rightarrow \pi=(0,1)^{*}\end{cases}
\end{gathered}
$$

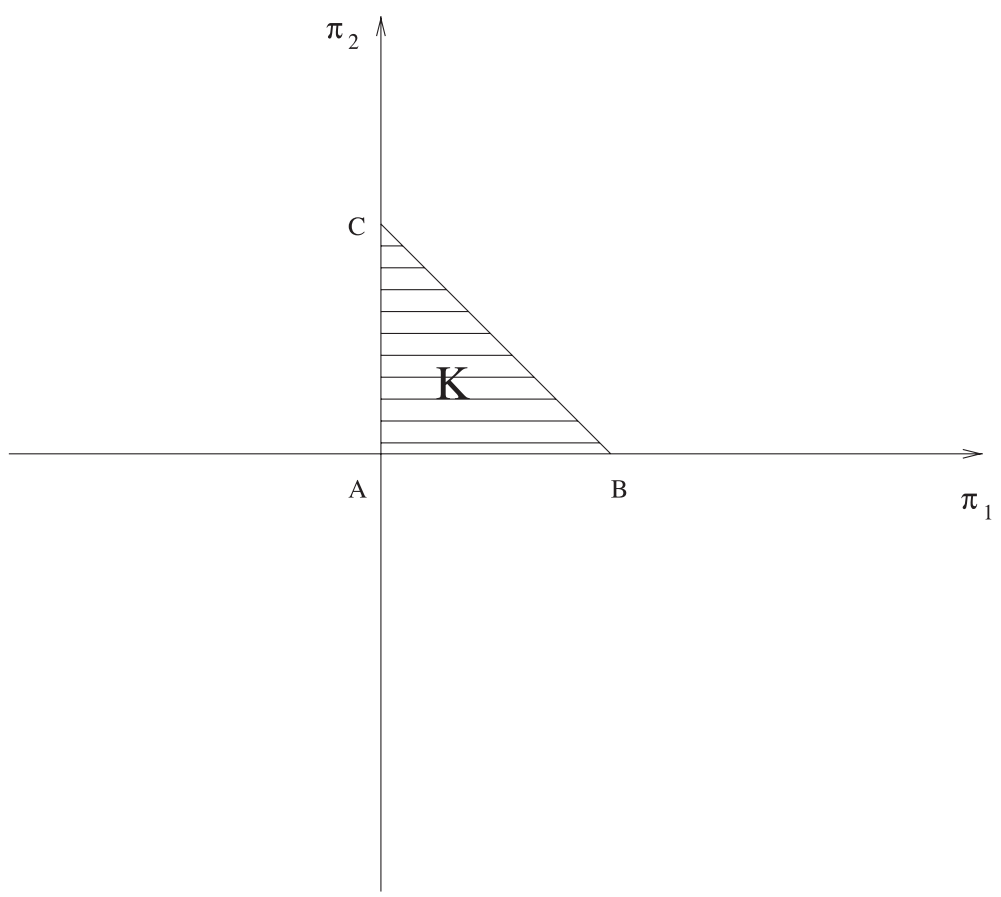

FIGURE 1. 


$$
\begin{aligned}
\sup _{\pi \in B C} f(\pi) & =\frac{\hat{\mu}_{1}\left(D_{22}-D_{12}\right)+\hat{\mu}_{2}\left(D_{11}-D_{12}\right)}{D_{11}+D_{22}-2 D_{12}} \\
& -\frac{\sqrt{\operatorname{det} D}}{D_{11}+D_{22}-2 D_{12}} \sqrt{\beta^{2}\left(D_{11}+D_{22}-2 D_{12}\right)-\left(\mu_{1}-\mu_{2}\right)^{2}}
\end{aligned}
$$

only if $\beta^{2} \geq\left(\mu_{1}-\mu_{2}\right)^{2} /\left(\left(D_{11}+D_{22}-2 D_{12}\right)\right)$. In this case the optimal strategy is

$$
\begin{aligned}
& \bar{\pi}_{1}= \frac{D_{22}-D_{12}}{D_{11}+D_{22}-2 D_{12}}+ \\
&+\frac{\left(\mu_{1}-\mu_{2}\right) \sqrt{\operatorname{det} D}}{D_{11}+D_{22}-2 D_{12}} \frac{1}{\sqrt{\beta^{2}\left(D_{11}+D_{22}-2 D_{12}\right)-\left(\mu_{1}-\mu_{2}\right)^{2}}} \\
& \bar{\pi}_{2}=1-\bar{\pi}_{1} .
\end{aligned}
$$

If the inner region of $K$ is denoted with $\stackrel{\circ}{K}$, the problem is similar to the unconstrained case analyzed in the previous subsection:

$$
\sup _{\pi \in K} f(\pi)=0
$$

if and only if $\beta \geq \sqrt{\hat{\mu} \cdot D^{-1} \hat{\mu}} \equiv\|q\|$. The corresponding optimal strategy is

$$
\pi_{2}=\left[\frac{\hat{\mu}_{2} D_{11}-\hat{\mu}_{1} D_{12}}{\hat{\mu}_{1} D_{22}-\hat{\mu}_{2} D_{12}}\right] \pi_{1}
$$

if $\beta=\|q\|$ and

$$
\pi=0
$$

if $\beta>\|q\|$.

Supposing, without losing in generality, that $\beta_{1}=\hat{\mu}_{1} / \sqrt{D_{11}}$ is less than $\beta_{2}=$ $\hat{\mu}_{2} / \sqrt{D_{22}}$, it can be shown that $\beta_{2}<\|q\|$. Let $\beta_{c}$ be the solution of the equation

$$
\sup _{\pi \in A C} f(\pi)=\sup _{\pi \in B C} f(\pi)
$$

or

$$
\begin{aligned}
\hat{\mu}_{2}-\beta_{c} \sqrt{D_{22}} & =\frac{\hat{\mu}_{1}\left(D_{22}-D_{12}\right)+\hat{\mu}_{2}\left(D_{11}-D_{12}\right)}{D_{11}+D_{22}-2 D_{12}}+ \\
& -\frac{\sqrt{\operatorname{det} D}}{D_{11}+D_{22}-2 D_{12}} \sqrt{\beta_{c}^{2}\left(D_{11}+D_{22}-2 D_{12}\right)-\left(\mu_{1}-\mu_{2}\right)^{2}}
\end{aligned}
$$




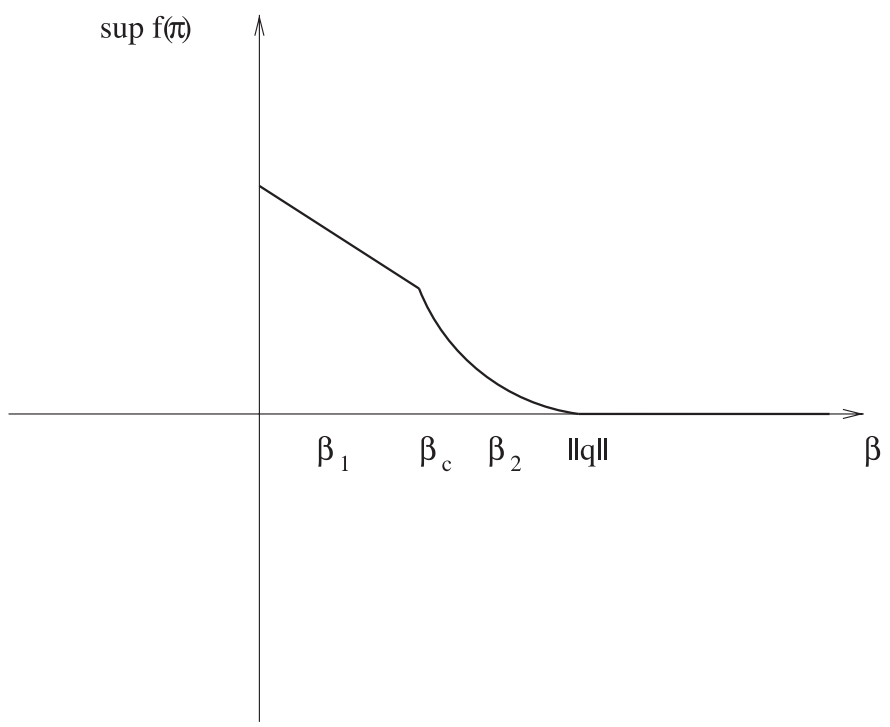

FIGURE 2.

The following relation holds:

$$
\frac{\left(\hat{\mu}_{1}-\hat{\mu}_{2}\right)^{2}}{D_{11}+D_{22}-2 D_{12}}<\beta_{c}<\beta_{2} .
$$

The analysis of equations (16-23) can be summarized as follows (see also figure 2):

- for $0<\beta<\beta_{c}$

$$
\sup _{\pi \in K} f(\pi)=\sup _{\pi \in A C} f(\pi)
$$

because clearly $\sup _{\pi \in A C} f(\pi)>\sup _{\pi \in A B} f(\pi)$ and in the inner points of $B C$ and $K$ the function $f(\pi)$ does not admit maxima. In such case the optimal strategy is $\pi=(0,1)^{*}$ : the agent invests all his wealth in asset $P_{2}$

- for $\beta=\beta_{c}$

$$
\sup _{\pi \in K} f(\pi)=\sup _{\pi \in A C} f(\pi)=\sup _{\pi \in B C} f(\pi)
$$

since in $\stackrel{\circ}{K}$ the function $f(\pi)$ does not admit maxima and $\sup _{\pi \in A C} f(\pi)>$ $\sup _{\pi \in A B} f(\pi)$. In this case $\pi$ is given by equations $(19,20)$ : the agent begins to invest also in asset $P_{1}$ but not in the risk-free asset

- for $\beta_{c}<\beta<\|q\|$

$$
\sup _{\pi \in K} f(\pi)=\sup _{\pi \in B C} f(\pi)
$$


since $\sup _{\pi \in B C} f(\pi)>\sup _{\pi \in A C} f(\pi)>\sup _{\pi \in A B} f(\pi)$ and in $\stackrel{\circ}{K}$ the function $f(\pi)$ does not admit maxima. The optimal strategy is given in equations $(19,20)$ : the agent invests in asset $P_{1}$ and $P_{2}$, and not in the risk-free asset

- for $\beta=\|q\|$

$$
\sup _{\pi \in K} f(\pi)=\sup _{\pi \in A C} f(\pi)=\sup _{\pi \in K} f(\pi)=0,
$$

since $\sup _{\pi \in A C} f(\pi)>\sup _{\pi \in A B} f(\pi)$. The optimal strategy is given by equation (22) or, equivalently, by $\pi=(0,0)^{*}$

- for $\beta>\|q\|$

$$
\sup _{\pi \in K} f(\pi)=\sup _{\pi \in K} f(\pi)
$$

since $\sup _{\pi \in A B} f(\pi)<\sup _{\pi \in A C} f(\pi)<\sup _{\pi \in B C} f(\pi)<0$ and $\sup _{\pi \in \hat{K}} f(\pi)=0$ in correspondence of the optimal strategy $\pi=(0,0)^{*}$.

In the last two cases $\sup _{\pi \in K} f(\pi)=0$ and repeating the same argument of equations (9-13) it can be argued that from the point of view of an agent with $\beta \geq\|q\|$, taking the supremum value of the final wealth over the Wang-distorted probability function is equivalent to taking the expected value of the final wealth in a risk-neutral world.

The result obtained in this subsection as well as the analysis of the unconstrained market case, allows to interpret the Wang parameter $\beta$ as a subjective estimate of the market price of risk: if $\beta$ is sufficiently small, $0<\beta<\|q\|$, the agent is "optimist" and invests all his wealth in the risky asset (in $P_{2}$ if $0<$ $\beta<\beta_{c}$ ) because his market price of risk perception $\beta$ is less than the actual one; when his estimate $\beta$ is equal to the actual market price of risk he invests indifferently in the risk-free asset or in an equivalent basket of risky assets. Finally, if $\beta>\|q\|$, the agent is "pessimist": his market price of risk estimate is too high than the actual one: in this case the agent invests his wealth in the risk-free asset only.

\subsection{A Comparison Between DEU and EU Solution}

In the classical EU theory the solution of the dynamic portfolio selection problem is determined by maximizing the expected terminal wealth utility $E\left[U\left(X_{T}^{t, x, \pi}\right)\right]$ with respect to variations in $\pi$, where $U: \mathbb{R}^{+} \rightarrow \mathbb{R}$ is usually a strictly increasing, strictly concave and twice differentiable utility function representing the preferences of a risk-averse agent.

If the optimization problem is unconstrained, a solution can be found by dynamic programming techniques which for given utility functions lead to closed form solutions. If the problem is constrained, convex analysis and duality theory can be applied; however, relatively simple explicit solutions are only 
available in very special cases ${ }^{6}$. The unconstrained problem is therefore more suitable for a direct comparison between the DEU solution using Wang transform and the EU theoretical solution.

In order to do such a comparison the agent utility function must be specified. If a logarithmic utility function $U(X)=\ln X$ or a power utility function $U(X)=X^{\gamma}, \gamma \in(0,1)$, is considered, the EU closed form solution for the unconstrained problem are respectively ${ }^{7}$

$$
\bar{\pi}=D^{-1} \hat{\mu}
$$

or

$$
\bar{\pi}=\frac{1}{1-\gamma} D^{-1} \hat{\mu}
$$

By comparing the solution for $\beta=\sqrt{\hat{\mu} \cdot D^{-1} \hat{\mu}}$ in subsection $5.1, \bar{\pi}=c D^{-1} \hat{\mu}$, $c \geq 0$, with equations (24) and (25), it follows that if respectively $c=1$ or $c=$ $\frac{1}{1-\gamma}$, the optimal DEU solution using the Wang transform is equal to the optimal EU solution. Since the logarithmic utility function and the power utility function form the constant relative risk-aversion (CRRA) utility function class, it can be inferred that the DEU solution of the unconstrained problem using the Wang transform is a generalization of the EU solution when the agent has a CRRA utility function.

\section{Final Remarks}

In this paper the dynamic portfolio selection problem has been studied for the first time in a DEU framework. Furthermore closed form solutions to the problem both with and without short-selling allowed have been provided by using the Wang transform as distortion function. It has been shown that the Wang transform can be used as an effective tool in this framework and that the resulting optimal portfolios vary according to the relation between the market price of risk and the Wang parameter. In particular if the Wang parameter is greater than or equal to the market price of risk the optimal portfolios expected return equals the risk-free rate. Finally it has been shown that the DEU solution of the unconstrained problem using the Wang transform can be considered a generalization of the EU solution when the agent has a CRRA utility function.

This paper can be considered a first step toward the application of the DEU theory to the asset pricing problem in incomplete markets and toward a new definition of the concept of "fair price".

\footnotetext{
6 For more details see Cvitanic-Karatzas (1992).

7 For more details see Merton (1990).
} 


\section{REFERENCES}

Allais, M. (1953) Le comportement de l'homme rationnel devant le risque: Critique des postulats et axiomes de l'école américaine. Econometrica 21, 503-546.

Cvitanic, J. and Karatzas, I. (1992) Convex Duality in Constrained Portfolio Optimization. Annals of Applied Probability 2, 767-818.

EllsberG, D. (1961) Risk, Ambiguity, and the Savage Axioms. Quarterly Journal of Economics 75, 643-669.

Hadar, J. and Kun Seo, T. (1995) Asset Diversification in Yaari's Dual Theory. European Economic Review 39, 1171-1180.

Hamada, M., Sherris, M. and van der Hoek, J. (2006) Dynamic Portfolio Allocation, the Dual Theory of Choice and Probability Distortion Function. ASTIN Bulletin 36, 187-217.

Karatzas, I. and Kou, S. (1996) On The Pricing Of Contingent Claims Under Constraints. Annals of Applied Probability 6, 321-369.

Merton, R. (1969) Lifetime Portfolio Selection under Uncertainty: The Continuous-Time Case. Review of Economics and Statistics 51, 247-257.

Merton, R. (1990) Continuous-Time Finance. Basil Blackwell, Oxford.

Quiggin, J. (1993) Generalized Expected Utility Theory: The Rank-Dependent Expected Utility Model. Kluwer-Nijhoff, Amsterdam.

Von Neumann, J. and Morgenstern, O. (1944) Theory of Games and Economic Behavior. Princeton University Press, Princeton.

WANG, S. and Young, V. (1998) Ordering risks: expected utility versus Yaari's dual theory of choice under risk. Insurance: Mathematics \& Economics 22, 145-162.

WANG, S. (2000) A Class of Distortion Operators for Pricing Financial and Insurance Risks. Journal of Risk and Insurance 67, 15-36.

WANG, S. (2002) A Universal Framework for Pricing Financial and Insurance Risks. ASTIN Bulletin 32, 213-234.

YAARI, M. (1987) The dual theory of choice under risk. Econometrica 55, 95-115.

Andrea GHeno

Università degli studi Roma Tre

Dipartimento di Economia

Via Silvio D’Amico 111

00145, Roma, Italy

E-mail: gheno@uniroma3.it 